\title{
Spatially Varying Mass Function of MACHOs in the Galactic Halo and Interpretation of Microlensing Results
}

\author{
Sohrab Rahvar \\ Department of Physics, Sharif University of Technology, P.O.Box 11365-9161, Tehran, Iran, \\ email:rahvar@sharif.edu \\ Institute for Studies in Theoretical Physics and Mathematics, P.O.Box 19395-5531, Tehran, \\ Iran
}

\begin{abstract}
The gravitational microlensing experiments in the direction of Large Magellanic Cloud (LMC) predict a large amount of white dwarfs $(\sim 20 \%)$ filling the galactic halo. However, the predicted white dwarfs have not been observed at the galactic halo. To interpret the microlensing results and resolving the mentioned problems, we use the hypothesis of spatially varying mass function of MACHOs, proposed by Kerins \& Evans (1998) (hereafter KE). However the KE model is not compatible with the duration distribution of events (Rahvar 2004a). Here we use more realistic power-law model of MF, $d n / d m \propto m^{\alpha}$ for the MACHOs of halo. The index of MF in this model changes from -2.7 for stars with $m>1 M_{\odot}$ at the central part of galactic halo to the substellar regime with an upper limit of -1 at the edge of halo. We show that in contrast to the abundant brown dwarfs of galactic halo, heavy MACHOs can be responsible for the microlensing events in the direction of LMC.
\end{abstract}

\section{Introduction}

The rotation curves of spiral galaxies and the Milky Way show that these type of galaxies have dark halo component. The most trivial candidate for the dark halo structure is the baryonic matter that can be in the form of gaseous or MAssive Compact Halo Objects (MACHOs). Since MACHOs are expected to be too light to be luminous and difficult detectable, Paczyński (1986) proposed an indirect method so-called gravitational microlensing to observe them indirectly. Following his suggestion several experiments such as EROS and MACHO started monitoring millions of LMC stars for one decade and observed less than 20 events in this direction (Alcock et al. 2000, Lasseree et al. 2000). Due to the degeneracy nature of gravitational microlensing problem, it is impossible to obtain the mass, distance and transverse velocity of the lenses by measuring the duration of events. The only way is using statistical studies, comparing the microlensing events with the models. The outcome of this study is the mean mass of MACHOs and their mass contribution in the galactic halo. For the standard Galactic model with the Dirac-Delta mass function (MF), halo is comprised by $20 \%$ of MACHOs with the mass of $0.5 M_{\odot}$. Comparing the luminous mass of Milky Way with that of halo, we can conclude that the white dwarfs of halo should have twice mass of the ordinary stars of the disk and bulge. KE proposed using Dirac Delta spatially varying MF as a solution to this problem but it has been shown that the duration distribution in this model is not compatible with the LMC microlensing candidates. Rahvar (2004a) used a likelihood analysis to find the best parameters for the model. Here we use more realistic MF for the MACHOs of halo as the power law function where the mass scale and the index of MF changes monotonically from the center to the edge of galaxy. We show that duration distribution of events in this 
model is compatible with the LMC microlensing candidates. The effective mean mass of lenses, as the mean mass of observed lenses in this model is larger than the mean mass of overall lenses of the galactic halo. This means that the galactic halo with the dominant brown dwarfs can produce microlensing events compatible with the observed data.

The organization of paper is as follows: In Section 2 we introduce the hypothesis of spatially varying MF of KE and power-law models. In Section 3 we compare the LMC microlensing events of MACHO experiment with the expected distribution from the model and section 4 contains the conclusion of this work.

\section{Spatially Varying MF}

The tradition in the microlensing analysis is using Dirac-Delta MF for the MACHOs of galactic halo. This function has been used for the sake of simplicity to apply it to an arbitrary MF of halo. However this approach hides the information about the contribution of the MACHOs with various masses in the gravitational microlensing candidates. Star and baryonic cluster formation theories (e.g., Ashman 1990; Carr 1994; De Paolis et al. 1995) predict the variation of MF in interstellar medium depending on the density of clouds forming the stars. Shadmehri (2004) and Elmegreen (2004) also showed that instead of universal initial mass function we can have a composite with various contributions from the brown dwarfs, solar masses and high mass stars, depending on the density of environment. Since the halo density changes as $\sim 1 / r^{2}$, we can conclude that the MF of MACHOs may change by distance from the center of galaxy. The inner halo comprises partly visible stars, in association with the globular cluster population, while the outer halo comprises mostly low-mass stars and brown dwarfs.

The hypothesis of using spatially varying MF has been proposed by KE. They used a spatially varying $\mathrm{MF}$ as

$$
M F(r)=\delta[M-M(r)]
$$

where the mass scale in this model decreases monotonically as a function of $r$ as

$$
M_{U}\left(\frac{M_{L}}{M_{U}}\right)^{r / R_{\text {halo }}}
$$

$M_{U}$ and $M_{L}$ are the upper and lower limits for the mass of MACHOs and $R_{\text {halo }}$ is the size of galactic halo. In the CMD plus baryonic halo models, $R_{\text {halo }}$ the size of halo which contains MACHOs can be extended more than the halo size.

Here we use more realistic spatially varying MF than the KE model. The MF of stars is taken as the power low function $d n / d m \propto m^{\alpha}$, where the index of function depends on the mass scale of stars. The upper limit of index (Chabrier 2002) for the substellar regime can be -1 and the lower limit (Scalo 1986) of -2.7 for the stars massive than the solar mass.

Here we use the following anzats for the spatially varying MF:

$$
\frac{d n}{d m}=\left[\frac{m}{m_{0}(r)}\right]^{\alpha(r)},
$$

where $m(r)>m_{0}(r)$ and the index of power-law changes as $\alpha(r)=1.7 \frac{r}{R_{\text {halo }}}-2.7$. The typical mass of MACHOs, $m_{0}(r)$ changes as $M_{U}\left(\frac{M_{L}}{M_{U}}\right)^{r / R_{\text {halo }}}$. Here we take the $M_{U}=1 M_{\odot}, M_{L}=0.001 M_{\odot}$ and $R_{\text {halo }}=100 \mathrm{kpc}$. Figure 1 shows the distribution of mass of MACHOs as a function of distance from the center of galaxy. 

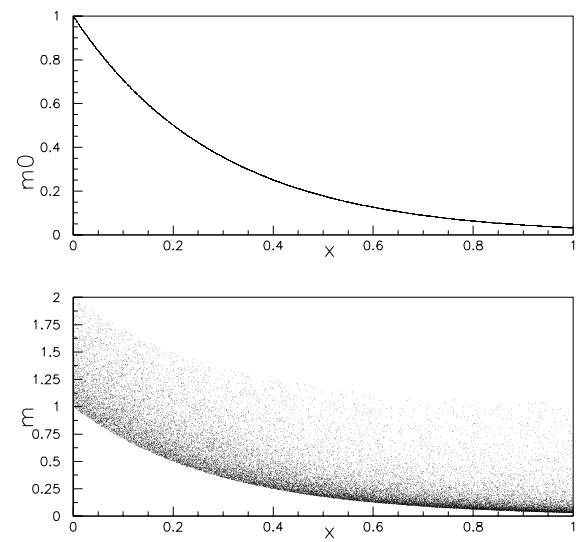

Figure 1. The mass distribution of MACHOs as a function of $x=D_{o l} / D_{o s}$, where $D_{o l}$ and $D_{o s}$ are the observer-lens and observer-source distances. The upper panel and lower panels show the distributions of $m_{0}$ and mass of MACHOs as function of distance.

\section{Microlensing Analysis in the Spatially Varying MF}

Here in this section our aim is to compare the microlensing data with the theoretical model. First we calculate the theoretical and experimental optical depths (Alcock et al. 2000) and compare them with each others. Using the experimental efficiency of observation, the observed optical depth obtain as follows:

$$
\tau_{o b s}=\frac{\pi}{4 E} \Sigma t_{i}
$$

where $t_{i}$ is the duration of ith event and $E$ is the exposure time of observation which for the 5.7 yrs observation of LMC by MACHO experiment, $E$ is $6.12 \times 10^{7}$. The optical depth results from $13 \mathrm{LMC}$ microlensing candidates is about $4.43 \times 10^{-8}$. One the other hand, using the power-law MF for the MACHOs of galactic halo in the standard halo model, we can produce the rate of microlensing events $\frac{d \Gamma}{d t}$. The theoretical optical depth is calculated as

$$
\tau_{\text {expected }}=\frac{\pi}{4} \int \frac{d \Gamma}{d t} \epsilon(t) t d t
$$

where we consider hundred percent of halo is made by MACHOs and $\epsilon(t)$ is the observational efficiency. The ratio of the observed to the theoretical optical depth results the MACHOs contribution in the galactic halo, $f=\frac{\tau_{\text {obs }}}{\tau_{\text {expected }}}$. Here in this model 23 percent of halo is made by MACHOs. Figure 2 shows the distribution of duration of events in the standard halo model, considering the observational efficiency of MACHO experiment.

We do a Monte-Carlo simulation to show quantitatively how the contribution of massive lenses in the microlensing events is more than the light ones. According to the distribution of matter along the line of sight the lenses are selected by the probability function as follows:

$$
\frac{d P}{d x} \propto \rho(x) \sqrt{x(1-x)}
$$

where $\rho$ is the matter density and $x$ is the lens-source to the observer-source distances. After selecting the position of lenses, we use the equation (2.3) to obtain their masses. The duration of events are generated through $t e=\frac{2 R_{E}}{v_{t}}$ and compared with the observational efficiency to be selected or rejected. $R_{E}$ is the Einstein radius of a lens and $v_{t}$ is its 


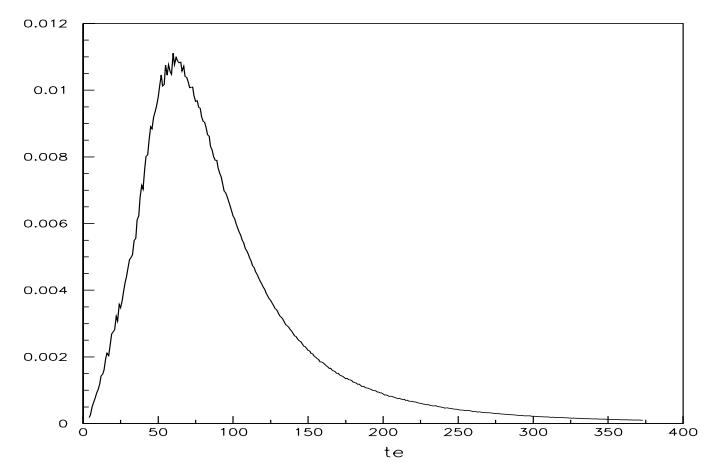

Figure 2. The duration distribution of LMC microlensing events $\left(t_{e}\right)$, considering the observational efficiency of MACHO experiment in Standard halo model and spatially varying power-law MF.

transverse velocity with respect to our line of sight. The mass of selected events are used to obtain the mean mass of lenses. We called the mean mass of these lenses as the active mean mass. In contrast to the active mean mass we define passive mean mass of lenses as the mean mass of overall lenses in the halo. The active and passive mean masses of lenses in this model are obtained as the 0.40 and 0.05 solar masses. This means that brown dwarfs are the abundant MACHOs of halo while white dwarfs are responsible to the microlensing events.

\section{Comparison with the observed data}

In this section we compare the expected duration distribution from the model with duration distribution of the observed LMC candidates. Two statistical parameters as the mean and the width of the duration distribution of events (Green and Jedamzik 2002; Rahvar 2004b) are used for our comparison. The width of the duration distribution for the $N_{o b s}$-th observed candidate is defined by

$$
\Delta t_{E}=\operatorname{Max}_{\left[j=1, N_{o b s}\right]}\left(t_{j}\right)-\operatorname{Min}_{\left[j=1, N_{o b s}\right]}\left(t_{j}\right) .
$$

The mean and the width of duration distribution of MACHO candidates are 97 and 188.2 days. Figure 3 compares the observed mean and width of duration of events with what we expect form the theoretical model. We perform a Monte-Carlo simulation to generate the mean and the width of distribution of events. Thirteen events is picked up from the duration distribution of events (Figure 2) and each time the mean and the width of events are calculated in each set to produce their distributions. Comparing these distributions with the observation show that the standard halo model with the power-law spatially varying $\mathrm{MF}$ is in good agreement with the LMC data.

\section{Conclusion}

In this work we use an anzats for the spatially varying power-law MF for the MACHOs of galactic halo. The advantage of using this MF is that (i) it is more realistic than DiracDelta MF, (ii) the expected duration distribution of events is in good agreement with the LMC microlensing data and (iii) the active mean mass of lenses as the mean mass of lenses contributing in the microlensing events obtained about $0.40 M_{\odot}$ compared to the passive mean mass of lenses as the mean mass of overall lenses of the galactic halo with 

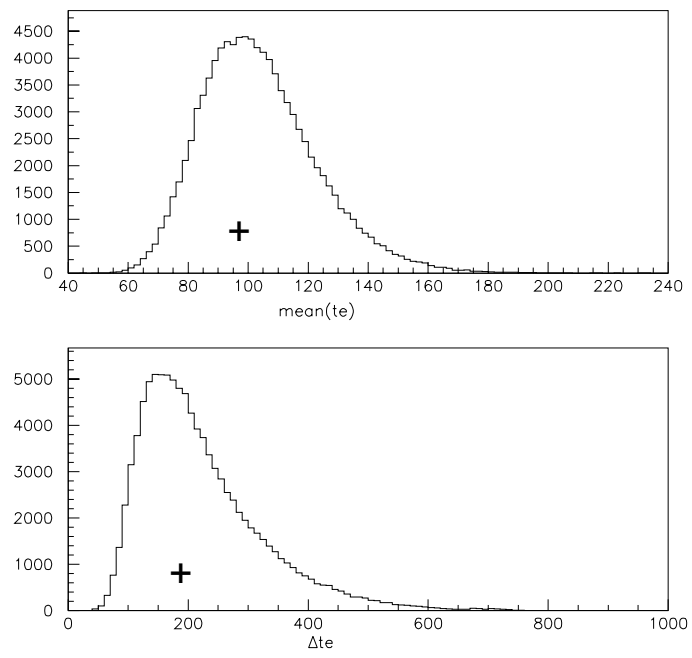

Figure 3. Upper and lower panels show the theoretical distributions of the mean and the width of microlensing events in standard halo model with spatially varying power-law MF. The cross signs are the mean and the width of duration of observed LMC candidates of MACHO experiment.

$0.05 M_{\odot}$. We showed that in a halo with abundant brown dwarfs, massive MACHOs with the white dwarf sizes can be responsible for the microlensing events that we are observing in the experiment.

\section{Acknowledgements}

I would like to say my thanks to the organizing committee of IAU225 and physics department of Sharif university, supporting me at this symposium.

\section{References}

Alcock C. et al. (MACHO) 2000, ApJ 542, 281

Ashman K. 1990, MNRAS 247, 662

Chabrier, G. 2002, ApJ 567, 304

Carr B. J. 1994, ARAEA 32, 531

De Paolis F., Ingrosso G., Jetzer P., \& Roncadelli M. 1995, A\&\&A 295, 567

Elmegreen, B. G. 2004, in press $M N R A S$ (astro-ph/0408231).

Green A. M., Jedamzik K. 2002, A\&A 395, 31

Kerins, E \& Evans N. W. 1998, ApJ 503, 75.

Lasserre T. et al. (EROS) 2000, A $6 A$ A55, L39.

Paczyński B. 1986, ApJ 304, 1.

Rahvar, S. 2004a, submitted in MNRAS(astro-ph/0404581).

Rahvar S. 2004, MNRAS 347, 213.

Scalo, J. M 1986, Fund. Cosm. Phys. 111.

Shadmehri, M. 2004, in press MNRAS(astro-ph/0407120).

\section{Discussion}

MARTin Dominik: How do things change if the unrealistic Dirac-Delta mass function is replaced by something appropriate? 
SOHRAB RAHVAR : This is a good idea and I checked it. I used a spatially varying powerlaw mass function as $d n / d m \propto m^{\alpha}$ for the MACHOs of halo. The index of MF in this model changes from -2.7 for stars with $m>1 M_{\odot}$ at the central part of galactic halo to the substellar regime with an upper limit of -1 at the edge of halo. 